\title{
Collecting core data in severely injured patients using a consensus trauma template: an international multicentre study
}

\author{
Kjetil Gorseth Ringdal ${ }^{1,2,3^{*}}$, Hans Morten Lossius ${ }^{1,4}$, J Mary Jones ${ }^{1,5}$, Jens M Lauritsen ${ }^{6,7}$, Timothy J Coats 8,9 , \\ Cameron S Palmer $^{10}$, Rolf Lefering ${ }^{11,12}$, Stefano Di Bartolomeo ${ }^{13,14}$, David J Dries ${ }^{15,16}$ and Kjetil Søreide ${ }^{4,17}$, for \\ The Utstein Trauma Data Collaborators
}

\begin{abstract}
Introduction: No worldwide, standardised definitions exist for documenting, reporting and comparing data from severely injured trauma patients. This study evaluated the feasibility of collecting the data variables of the international consensus-derived Utstein Trauma Template.

Methods: Trauma centres from three different continents were invited to submit Utstein Trauma Template core data during a defined period, for up to 50 consecutive trauma patients. Directly admitted patients with a New Injury Severity Score (NISS) equal to or above 16 were included. Main outcome variables were data completeness, data differences and data collection difficulty.

Results: Centres from Europe $(n=20)$, North America $(n=3)$ and Australia $(n=1)$ submitted data on 965 patients, of whom 783 were included. Median age was 41 years (interquartile range (IQR) 24 to 60), and $73.1 \%$ were male. Median NISS was 27 (IQR 20 to 38), and blunt trauma predominated (91.1\%). Of the 36 Utstein variables, 13 (36\%) were collected by all participating centres. Eleven (46\%) centres applied definitions of the survival outcome variable that were different from those of the template. Seventeen (71\%) centres used the recommended version of the Abbreviated Injury Scale (AIS). Three variables (age, gender and AIS) were documented in all patients. Completeness $>80 \%$ was achieved for 28 variables, and 20 variables were $>90 \%$ complete.

Conclusions: The Utstein Template was feasible across international trauma centres for the majority of its data variables, with the exception of certain physiological and time variables. Major differences were found in the definition of survival and in AIS coding. The current results give a clear indication of the attainability of information and may serve as a stepping-stone towards creation of a European trauma registry.
\end{abstract}

\section{Introduction}

Major trauma is a leading cause of death and disability around the world [1], and it accounts for approximately $10 \%$ of the world's deaths. Globally, unintentional injuries are ranked as the sixth leading cause of death and the fifth leading cause of moderate and severe disability [2]. The introduction of regionalised trauma systems has the potential to reduce preventable deaths [3], but an improved understanding of the benefits and limitations

\footnotetext{
* Correspondence: kjetil.ringdal@norskluftambulanse.no

'Department of Research, Norwegian Air Ambulance Foundation,

Holterveien 24, N-1440 Drøbak, Norway

Full list of author information is available at the end of the article
}

of different trauma care systems requires comparison across systems [4]. However, it has been shown that the datasets of existing trauma registries frequently lack compatible definitions of common data variables [5-9]. Consequently, the comparison and interpretation of trauma system outcomes has been hampered [10]. The lack of dataset uniformity poses substantial challenges to initiatives seeking to assess the quality of healthcare systems [11]. Several regions, particularly in North America, have implemented systematic documentation of trauma care and trauma system performance [12]. However, such documentation is limited in Europe [5,13,14], where no joint trauma registry exists $[5,15]$.

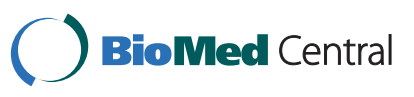


A recent European collaboration (the EuroTARN Group) assessed the potential of creating a data collection trial among a number of trauma registries in Europe, and the potential for comparing summary data and crude mortality rates [5]. Due to differences between trauma registries, the collaboration recognised that meaningful outcome comparisons were not possible. Similar conclusions were also reached in a contemporary Scandinavian report [9].

To address the discrepancies raised in these reports, members of the German, Italian, Scandinavian and UK trauma registries $[15,16]$ performed an expert panel consensus process to develop a core set of uniform patientlevel data for documenting and reporting trauma incidents. The resulting template, the Utstein Trauma Template [15-17], consists of recommended eligibility criteria and a set of 36 core data variables with four subsidiary variables.

The aim of the current study was to evaluate the feasibility of collecting patient-level data for severely injured patients across trauma centres using the Utstein Trauma Template variables as a standard.

\section{Materials and methods Study design}

The study was a prospective international multicentre feasibility study, in which each participating institution was asked to collect and code up to 50 consecutively hospitalised trauma patients during the study period. The reporting of this study aims at conforming to the STROBE statement for reporting observational studies [18].

\section{Participants}

The primary focus was on inviting trauma registries from a mix of small, medium and large volume European trauma centres. However, to ensure that a degree of valid worldwide comparability was assessed, centres from North America and Australia were also invited.

Trauma centres were invited using a standardised open letter sent by email. For centres that agreed to participate, three reminder emails were sent to those that had not submitted data within the deadline. No followup was performed for the institutions that did not respond to the first invitation letter.

\section{Patients}

Trauma centres were asked to include directly and consecutively admitted trauma patients with a New Injury Severity Score (NISS) [19] $\geq 16$ who presented between 1 September 2009 and 30 November 2009. Patients were excluded if they were transferred to the hospital, admitted to the hospital $>24$ hours after injury, or if they were declared dead before hospital arrival or with no signs of life upon hospital arrival and no response to initial hospital resuscitation. Patients with asphyxia or drowning injuries and patients who had burns as the predominant injury were also excluded [16].

\section{Data variables}

Participants were asked to collect all the data variables of the Utstein Trauma Template [16] and to fill out and return a self-administered questionnaire (Additional file 1 ). Using the questionnaire, the centres were asked to report the data variables that they were able to collect, whether their data variable definitions deviated from the definitions of the template, and if they experienced any data collection difficulties. Additional comments could be made for each variable. The centres were asked to grade all injuries according to the Abbreviated Injury Scale (AIS) 2005 or 2005-update 2008 [20], reporting the whole seven digit AIS code.

The main outcome measures were the data completeness of information at the patient and variable levels, the discrepancy for data variable definitions, and the difficulty of data collection. The completeness of the patient-level data was measured on the basis of reported values, while unknown and blank values were considered as missing values. The discrepancy for definitions and the collection difficulty were assessed from the associated questionnaire and were based on "yes", "no" and "unknown" answers.

\section{Data collection}

Patient-level data were collected using the local hospitalbased or regional trauma registries. For participants without a suitable registry, an electronic database was provided by the investigators. Centres that did not return the questionnaires, provide the patient-level data, or return a consent form were excluded from the study.

\section{Study size}

The sample size of 50 cases per centre was chosen as a pragmatic size to balance the workload imposed by the study while providing a reasonable number of patients for computing completeness proportions. Low-volume trauma centres that were not able to collect 50 cases within the timeframe were asked to submit three months of hospitalised patients.

\section{Ethics}

The study was approved by the Regional Committee for Medical and Health Research Ethics of Southeast Norway and the Norwegian Social Sciences Data Services. The exported datasets were required to not contain any direct or indirect patient identifiable data. Dates and times were not permitted in the submitted datasets, and patients could not be marked with a reference number 
that could be linked to a patient number from the submitting centre. The centres were required to return a written and signed consent form stating that the participation and data sharing was in compliance with their own specific institutional and/or national legal frameworks and data protection requirements.

\section{Statistical methods}

Continuous data that were not normally distributed are presented using median and interquartile range (IQR) and analysed using non-parametric techniques. Categorical data are presented as counts and proportions. The completeness of the Utstein core data variables are presented as counts and proportions with 95\% confidence intervals (using Wilson's method [21]), and the completeness of each dataset was judged by the number of centres with complete patient data using percentile levels $\left(50^{\text {th }}, 75^{\text {th }}\right.$ and $\left.100^{\text {th }}\right)$. The desired goal of data completeness was set at $\geq 80 \%$.

Data were analysed using IBM SPSS version 18 (IBM Company, Chicago, IL, USA) and Stata/SE version 11.1 (StataCorp LP, College Station, TX, USA).

\section{Results}

\section{Participating centres}

Of the 42 centres invited, 10 never responded, four centres declined to participate due to resource constraints, and four centres agreed to participate but never submitted the requested material. Twenty-four of the invited centres (57\%) participated in the study (Figure 1), of which nine had been part of the Utstein Template development process.

In total, 14 nations were represented. Participation amongst Scandinavian invitees was 64\%, participation amongst European invitees outside Scandinavia was $61 \%$, and participation amongst North American/Australian centres was $40 \%$.

Two participants were large, multi-institutional trauma registries that represented collaborations of hospitals (152 and 120 hospitals, respectively), and 20 participants were individual hospitals with a hospital-based registry (Table 1 ). Two participants did not have a registry prior to the initiation of the study. The two multinational trauma registries and eight centres with a hospital-based registry had fully or partially implemented the Utstein variables prior to the initiation of the study. The remaining 14 centres only collected the Utstein data for the current study.

Twenty-two of the participating centres submitted data from trauma patients who were consecutively admitted, while the two multicentre trauma registries submitted data from patients who were consecutively included in their trauma registries.

\section{Patient characteristics}

In total, data from 965 patients were submitted. Of these, 182 (19\%) were excluded for not meeting the study selection criteria (Figure 1). AIS codes were missing for 12 patients, 94 patients had a NISS $<16$, and 76 patients were transferred to the reporting hospital. Therefore, $783(81 \%)$ patients were available for analyses, with 623 (80\%) patients from European centres.

Patient characteristics are summarised in Table 2. The majority of the patients were male $(73.1 \%)$, and the median age was 41.0 years (IQR 24 to 60 ). Blunt trauma predominated $(91.1 \%)$, while traffic accidents $(53.1 \%)$ and high-energy falls (19.3\%) were the most prominent injury mechanisms. The median NISS was 27.0 (IQR 20 to 38 ), and the reported death rate was $14.0 \%$.

\section{Data variables collected by centres}

Of the 36 Utstein variables, 13 (36\%) variables were collected in all 24 centres (Table 3). The variable that was recorded by the fewest centres was "Time Until Normal Arterial Base Excess", which was recorded by 17 participants $(70.8 \%)$ with a completion level of $48.2 \%$ (Figure 2 and Additional file 2). Of all the Utstein variables, four (11\%) variables did not deviate from the template's definitions in any of the centres (Table 3). Several centres had data variable definitions that differed from the definitions of the Utstein Template. The most heterogeneously defined variable was "Survival Status" (the Utstein recommendation is outcome at Day 30 after injury [11]), and $11(46 \%)$ centres used different definitions (Table 3): six used outcome at end of acute care stay, three used the in-hospital 30-day outcome, and two used the outcome at the end of total somatic stay (including rehabilitation). All centres used the AIS system for anatomical severity scoring. However, only 17 (71\%) of the centres used the versions recommended. Two centres submitted the single-digit AIS severity codes, excluding the six-digit injury descriptor.

Only two variables, "Gender" and "Age", were collected from all centres without difficulty. The variable that was most frequently reported to be difficult to collect was "Pre-Hospital Respiratory Rate", which eight centres (35\%) reported as difficult (Table 3).

\section{Completeness of patient-level core data}

The levels of completion for each of the Utstein variables are shown in Figure 2 and Additional file 2. Some centres declined to record specific variables but nevertheless submitted data on those variables for some patients. After exclusion of these datasets, the results showed that 20 Utstein core variables were $>90 \%$ complete. Of these, three variables (age, gender, and AIS) were $100 \%$ complete. Twenty-eight data variables were > 


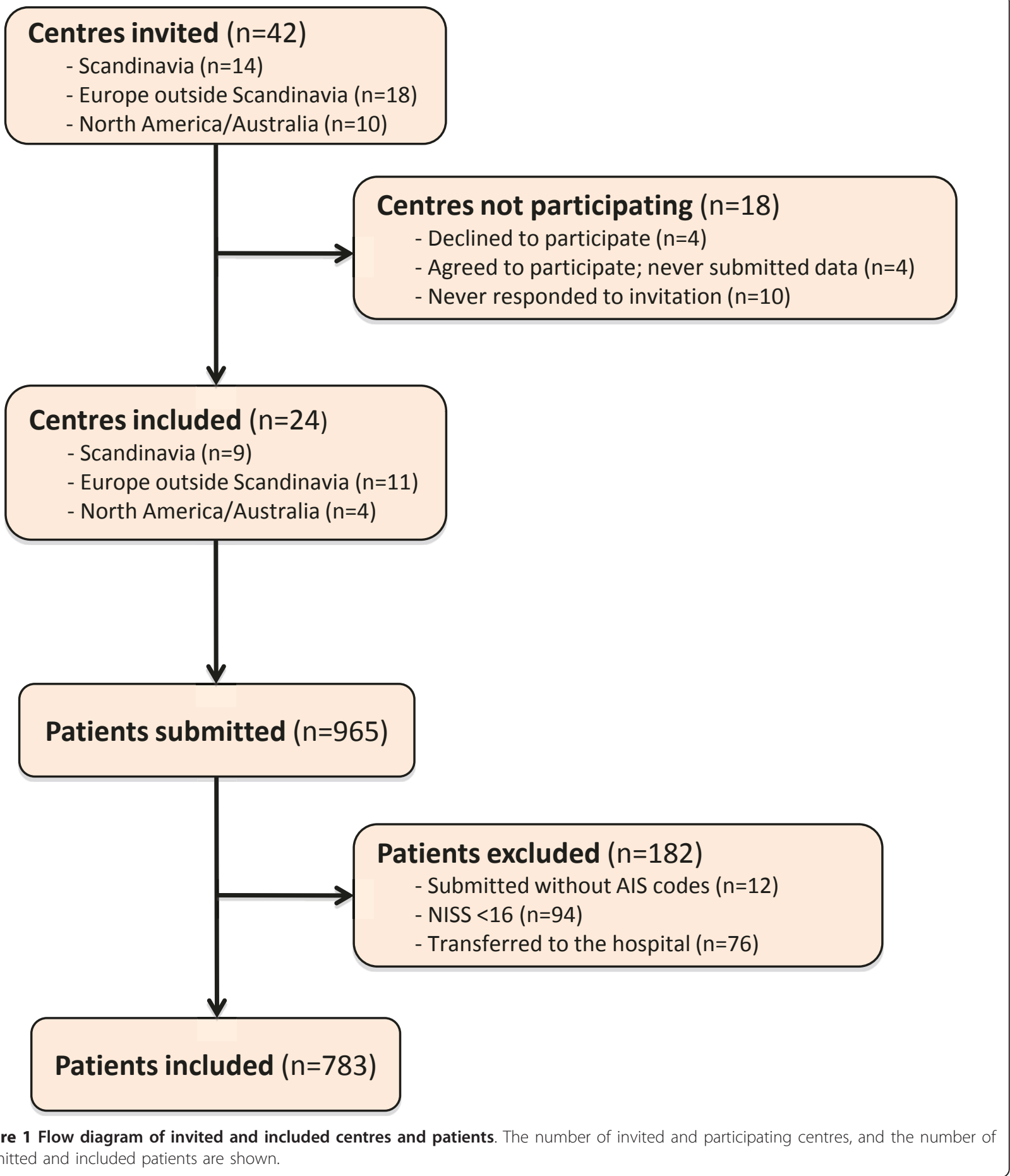

$80 \%$ complete. Eight variables had completeness levels that were below the desired $80 \%$ threshold (Figure 2). The variables "Time Until Normal Arterial Base Excess", "Arterial Base Excess", and "Pre-Hospital Respiratory Rate", had the lowest levels of completeness.

For reporting pre- and in-hospital SBP and RR values, the Utstein Template recommends the use of clinical categories (based on the Revised Trauma Score (RTS) categories [22]) when continuous values are missing [15]. This is illustrated in the results presented in Figure 2 and Additional file 2. When the continuous and categorical values of pre-hospital SBP and RR were combined, the completeness increased by $8.9 \%$ and $23.2 \%$, respectively (Figure 3 ). The equivalent in- 
Table 1 Characteristics of participating centres $(n=24)$

\begin{tabular}{lc}
\hline Centre characteristics & Values \\
\hline $\begin{array}{l}\text { Data collection for the project, } \mathbf{n}(\%) \\
\quad \text { In own registry } \\
\quad \text { In database designed for purpose }\end{array}$ & $19(79.2 \%)$ \\
Utstein data variables implemented prior to study initiation, $\mathbf{n}(\%)$ & $5(20.8 \%)$ \\
$\quad$ Yes & $10(41.7 \%)$ \\
$\quad$ No & $14(58.3 \%)$ \\
Type of pre-study registry, $\mathbf{n}(\%)$ & $20(83.3 \%)$ \\
$\quad$ Local/regional & $2(8.3 \%)$ \\
$\quad$ National/multi-national & $2(8.3 \%)$ \\
$\quad$ No registry & $191(110$ to 490$) ; n=17$ \\
Number of patients with ISS > 15 received in 2008, median (IQR) & $200(78$ to 1794$) ; n=10$ \\
Number of patients with NISS > 15 in 2008, median (IQR) & $1(1$ to 152$)$
\end{tabular}

IQR, Interquartile range; ISS, Injury Severity Score; NISS, New Injury Severity Score.

hospital completeness levels showed an increase of $1.9 \%$ and $17.6 \%$.

\section{Discussion}

The current international multicentre study demonstrated acceptable feasibility and completeness in reporting trauma data using a common template. For the majority of variables, the data collection was sufficient, while some areas in need for improvement were identified. The feasibility of bearing this project to fruition may serve as a stepping-stone towards establishment of a common pan-European trauma registry. However, some results deserve further discussion.

This study demonstrated that the data for 28 (78\%) of the Utstein variables were $>80 \%$ complete, and that the data for $20(56 \%)$ variables were > 90\% complete. The pre-hospital SBP and RR values were less complete than were the equivalent in-hospital values. This result is consistent with findings from Arbabi et al. [23], who found that pre-hospital and admission SBP values were recorded for $35 \%$ and $67 \%$ patients, respectively. In cases with missing continuous values, the Utstein Template recommends documenting the SBP and RR values as RTS categories [15,16]. This recommendation is not merely a mathematical consideration; it has a practical sense because clinical categories can be reasonably approximated by palpation of the patient's pulses and by chest examination. In the present study, the combination of the continuous and categorical SBP and RR values resulted in increased completeness compared to the sole use of continuous values (Figure 3). Although categorising continuous data may result in loss of precision and power in addition to other methodological challenges [24,25], the use of the clinical categories provides an undeniable advantage over not having data.
All centres reported injuries according to the AIS system, although injury documentation standards varied. Even though the majority of participating institutions used the AIS dictionaries recommended, nearly 30\% did not. Several recent studies have identified differences between the AIS 1998 and 2005/2008 dictionaries in terms of the number of patients classified as 'major trauma' [26-28], illustrating that injury data collected using different AIS dictionaries cannot be directly compared. When comparing outcomes, Injury Severity Score (ISS) [29] or NISS values, AIS dictionary differences could affect the discrimination between severely and less severely injured patients across national and international registries. In light of the recent literature, it is not clear whether parallel coding using the AIS 1998 and AIS 2005/2008 versions should be recommended in order to enable comparisons. However, a solution to overcome the limitation of the existing mapping tool in the AIS dictionary [30] may be a newly developed AIS98 to AIS08 mapping tool [30].

The Utstein Template recommends the use of the short-term outcome variable '30-day survival', which is a mortality indicator that is also applied in other fields such as stroke and acute myocardial infarction [31]. The definitions of the survival outcome variable differed across the participating centres included in the current study. Some centres evaluated short-term outcome based on hospital administrative data, which resulted in the use of in-hospital survival or in-hospital 30-day survival. Others used 30-day survival regardless of whether the patient was still hospitalised. These differences may result in unfavourable biases when trauma care is compared. The use of in-hospital 30-day survival can be particularly problematic with short length of hospital stay or increased tendencies for transfer of patients between facilities. Thus, a greater proportion of deaths within 30 
Table 2 Characteristics of the included trauma patients $(n=783)$

\begin{tabular}{|c|c|}
\hline Demographics & Values \\
\hline Age, median (IQR) & 41 (24 to 60$)$ \\
\hline \multicolumn{2}{|l|}{ Gender, n (\%) } \\
\hline Male & $572(73.1 \%)$ \\
\hline Female & $211(26.9 \%)$ \\
\hline \multicolumn{2}{|l|}{ Dominating type of injury, $n$ (\%) } \\
\hline Blunt & $713(91.1 \%)$ \\
\hline Penetrating & $68(8.7 \%)$ \\
\hline Missing data & $2(0.2 \%)$ \\
\hline \multicolumn{2}{|l|}{ Mechanism of injury, $\mathrm{n}(\%)$} \\
\hline $\begin{array}{l}\text { Traffic: motor vehicle accident (excluding } \\
\text { motorcycle) }\end{array}$ & $154(19.7 \%)$ \\
\hline Traffic: motorcycle accident & $114(14.6 \%)$ \\
\hline Traffic: bicycle accident & $48(6.1 \%)$ \\
\hline Traffic: pedestrian & $68(8.7 \%)$ \\
\hline Traffic: other & $31(4.0 \%)$ \\
\hline Shot & $36(4.6 \%)$ \\
\hline Stabbed & $31(4.0 \%)$ \\
\hline Struck or hit by blunt object & $33(4.2 \%)$ \\
\hline Low energy fall & $87(11.1 \%)$ \\
\hline High energy fall & $151(19.3 \%)$ \\
\hline Other & $22(2.8 \%)$ \\
\hline Unknown & $5(0.6 \%)$ \\
\hline Missing data & $3(0.4 \%)$ \\
\hline \multicolumn{2}{|l|}{ Injuries grouped by AIS body regions, $\mathrm{n}$ (\%) } \\
\hline Head & $\begin{array}{c}1,148 \\
(26.4 \%)\end{array}$ \\
\hline Face & 407 (9.4\%) \\
\hline Neck & $16(0.4 \%)$ \\
\hline Thorax & $713(16.4 \%)$ \\
\hline Abdomen & $252(5.8 \%)$ \\
\hline Spine & $426(9.8 \%)$ \\
\hline Upper extremity & $388(8.9 \%)$ \\
\hline Lower extremity & $532(12.3 \%)$ \\
\hline External and other & $85(2.0 \%)$ \\
\hline Missing data & $375(8.6 \%)$ \\
\hline \multicolumn{2}{|l|}{ Injuries grouped by AIS severity levels, n (\%) } \\
\hline AIS 1 to 3 & $\begin{array}{c}3,554 \\
(81.9 \%)\end{array}$ \\
\hline AIS 4 to 6 & $772(17.8 \%)$ \\
\hline AIS 9 (unknown) & $16(0.3 \%)$ \\
\hline \multicolumn{2}{|l|}{ NISS groups, n (\%) } \\
\hline 16 to 24 & $313(40.0 \%)$ \\
\hline 25 to 40 & $283(36.1 \%)$ \\
\hline 41 to 56 & $123(15.7 \%)$ \\
\hline 57 to 75 & $64(8.2 \%)$ \\
\hline \multicolumn{2}{|l|}{ Survival status, $\mathbf{n}(\%)$} \\
\hline Died & $110(14.0 \%)$ \\
\hline Survived & $621(79.3 \%)$ \\
\hline Unknown & $43(5.5 \%)$ \\
\hline Missing data & $9(1.1 \%)$ \\
\hline
\end{tabular}

AIS, Abbreviated Injury Scale; IQR, Interquartile range; NISS, New Injury Severity Score days of injury may be missed if only 'in-hospital deaths' are considered [11,15,31-33]. The endpoints 'in-hospital survival' and '30-day survival' should both be considered included in the Utstein Template until the health care systems have matured to the point where data about 30day survival status are easily obtainable.

This study does have associated limitations. First, the process applied for identifying centres for invitation was subjective and not standardised. Participating centres may be more likely to comply with the Utstein Template or better able to collect and report data requested. Second, the 10 centres not responding to invitations and the 4 that agreed to participate but never submitted the requested materials were not further contacted. Thus, we cannot preclude the possibility that they found collection of the dataset too difficult or time consuming.

Third, some institutions had already integrated the Trauma Template variables in their trauma documentation protocols and registries prior to the start of the current study, while others only collected these data for the study. Implementation of the template across all centres should have yielded a higher degree of completeness for some data sets. Fourth, participation from North America and Australia was low. However, because of the formalised criteria with which trauma care in American centres is reviewed, there is a greater homogeneity among these centres and data collection. Thus, despite the small number of hospitals, inclusion of three leading centres from the United States gives a good sampling of North American practice. Fifth, the desired goal of completeness (> 80\%) used in this study is an arbitrarily chosen threshold. No justifications or guidelines for the acceptability of missing data in registry studies (for example, prognostic studies) exist [personal communication with Professor Douglas G. Altman, University of Oxford, UK]. Thus, the threshold value was a choice based on consensus among the authors. Finally, the template allows some data fields to be left blank when a data variable is unknown or not documented. Leaving a data field blank can make it more difficult to estimate the exact completeness or perform comparative analyses (that is, the exact cause of leaving a data field blank could be "not measured", "forgotten" or "unknown").

This study demonstrates that considerable support exists for the development of an international uniform mandatory core dataset that can be the basis of a European trauma registry. However, several steps still remain. The current Utstein Trauma Template variables and definitions could be further improved before collaborative research on the comparison of trauma care performance is initiated on a larger scale. Hopefully, the results from this study will contribute to improvements. Indeed, at the time of the development of the Utstein 
Table 3 Number and proportion of collected Utstein variables, differences in variable definitions, and data collection difficulties

\begin{tabular}{|c|c|c|c|}
\hline Core data variable & $\begin{array}{c}\text { Centres collecting this data } \\
\text { variable } \\
\text { n (\%) }\end{array}$ & $\begin{array}{c}\text { Applied a different } \\
\text { definition } \\
\mathrm{n}(\%) \\
\end{array}$ & $\begin{array}{c}\text { Data variable was difficult to } \\
\text { collect } \\
\mathrm{n}(\%)\end{array}$ \\
\hline \multicolumn{4}{|l|}{ Predictive model variables } \\
\hline Gender & $24(100 \%)$ & 0 & 0 \\
\hline In-hospital SBP & $24(100 \%)$ & 0 & $1(4.2 \%)$ \\
\hline Hospital length of stay & $24(100 \%)$ & 0 & $1(4.2 \%)$ \\
\hline Age & $24(100 \%)$ & $1(4.2 \%)$ & 0 \\
\hline Dominating type & $24(100 \%)$ & $2(8.3 \%)$ & $1(4.2 \%)$ \\
\hline Intention of injury & $24(100 \%)$ & $2(8.3 \%)$ & $3(12.5 \%)$ \\
\hline Discharge destination & $24(100 \%)$ & $4(16.7 \%)$ & $2(8.3 \%)$ \\
\hline Mechanism of injury & $24(100 \%)$ & 7 (29.2\%) & $3(12.5 \%)$ \\
\hline Abbreviated Injury Scale & $24(100 \%)$ & $8(33.3 \%)$ & $1(4.2 \%)$ \\
\hline Survival status & $24(100 \%)$ & $11(45.8 \%)$ & $4(16.7 \%)$ \\
\hline In-hospital RR & $23(95.8 \%)$ & 0 & $6(25.0 \%)$ \\
\hline Pre-hospital SBP & $23(95.8 \%)$ & $1(4.3 \%)$ & $5(21.7 \%)$ \\
\hline Pre-hospital GCS & $23(95.8 \%)$ & $2(8.7 \%)$ & $5(21.7 \%)$ \\
\hline Pre-hospital RR & $23(95.8 \%)$ & $2(8.7 \%)$ & $8(34.8 \%)$ \\
\hline Pre-hospital GCS motor component & $22(91.7 \%)$ & $2(9.1 \%)$ & $6(27.3 \%)$ \\
\hline In-hospital GCS & $22(91.7 \%)$ & $2(9.1 \%)$ & $1(4.5 \%)$ \\
\hline Pre-hospital cardiac arrest & $22(91.7 \%)$ & $3(13.6 \%)$ & $1(4.5 \%)$ \\
\hline Pre-injury ASA-PS classification & $22(91.7 \%)$ & $7(31.8 \%)$ & $6(27.3 \%)$ \\
\hline In-hospital GCS motor component & $21(87.5 \%)$ & $2(9.5 \%)$ & $1(4.8 \%)$ \\
\hline Days on ventilator & $21(87.5 \%)$ & $5(23.8 \%)$ & $5(23.8 \%)$ \\
\hline INR & $20(83.3 \%)$ & $3(15.0 \%)$ & $1(5.0 \%)$ \\
\hline GOS score at discharge & $20(83.3 \%)$ & $3(15.0 \%)$ & $5(25.0 \%)$ \\
\hline Pre-hospital SBP-clinical category ${ }^{a}$ & $19(79.2 \%)^{\mathrm{a}}$ & $1(5.3 \%)^{a}$ & $1(5.3 \%)^{a}$ \\
\hline Arterial base excess & 19 (79.2\%) & $1(5.3 \%)$ & $3(15.8 \%)$ \\
\hline In-hospital RR-clinical category ${ }^{a}$ & $18(75.0 \%)^{\mathrm{a}}$ & $2(11.1 \%)^{\mathrm{a}}$ & $2(11.1 \%)^{\mathrm{a}}$ \\
\hline Pre-hospital RR-clinical category ${ }^{\text {a }}$ & $18(75.0 \%)^{a}$ & $2(11.1 \%)^{a}$ & $4(22.2 \%)^{a}$ \\
\hline In-hospital SBP-clinical category ${ }^{\text {a }}$ & $17(70.8 \%)^{a}$ & $1(5.9 \%)^{\mathrm{a}}$ & $1(5.9 \%)^{\mathrm{a}}$ \\
\hline \multicolumn{4}{|l|}{ System characteristics } \\
\hline Inter-hospital transfer & $24(100 \%)$ & $1(4.2 \%)$ & $2(8.3 \%)$ \\
\hline Transportation type & $24(100 \%)$ & $4(16.7 \%)$ & $2(8.3 \%)$ \\
\hline Type of first key emergency intervention & $24(100 \%)$ & $4(16.7 \%)$ & $2(8.3 \%)$ \\
\hline Highest level of in-hospital care & $23(95.8 \%)$ & $1(4.3 \%)$ & $4(17.4 \%)$ \\
\hline Pre-hospital airway management & $23(95.8 \%)$ & $3(13.0 \%)$ & $4(17.4 \%)$ \\
\hline Trauma team activation & $22(91.7 \%)$ & $2(9.1 \%)$ & $3(13.6 \%)$ \\
\hline Time from alarm until hospital arrival & $22(91.7 \%)$ & $2(9.1 \%)$ & $5(22.7 \%)$ \\
\hline $\begin{array}{l}\text { Highest level of pre-hospital care } \\
\text { provided }\end{array}$ & $22(91.7 \%)$ & $4(18.2 \%)$ & $4(18.2 \%)$ \\
\hline $\begin{array}{l}\text { Type of pre-hospital airway } \\
\text { management }\end{array}$ & $20(83.3 \%)$ & $4(20.0 \%)$ & $4(20.0 \%)$ \\
\hline \multicolumn{4}{|l|}{ Process mapping data } \\
\hline Time until first CT scan & $23(95.8 \%)$ & $4(17.4 \%)$ & 7 (30.4\%) \\
\hline Time from alarm until arrival at scene & $22(91.7 \%)$ & $1(4.5 \%)$ & $6(27.3 \%)$ \\
\hline $\begin{array}{l}\text { Time until first key emergency } \\
\text { intervention }\end{array}$ & $22(91.7 \%)$ & $4(18.2 \%)$ & $4(18.2 \%)$ \\
\hline Time until normal arterial base excess & $17(70.8 \%)$ & $2(11.8 \%)$ & $6(35.3 \%)$ \\
\hline
\end{tabular}

${ }^{a}$ The clinical categories relate to cases for which continuous data were not submitted.

ASA-PS, American Society of Anesthesiologists Physical Status; EMS, emergency medical service; GCS, Glasgow Coma Scale; GOS, Glasgow Outcome Scale; LOS, length of stay; RR, respiratory rate; SBP, systolic blood pressure 


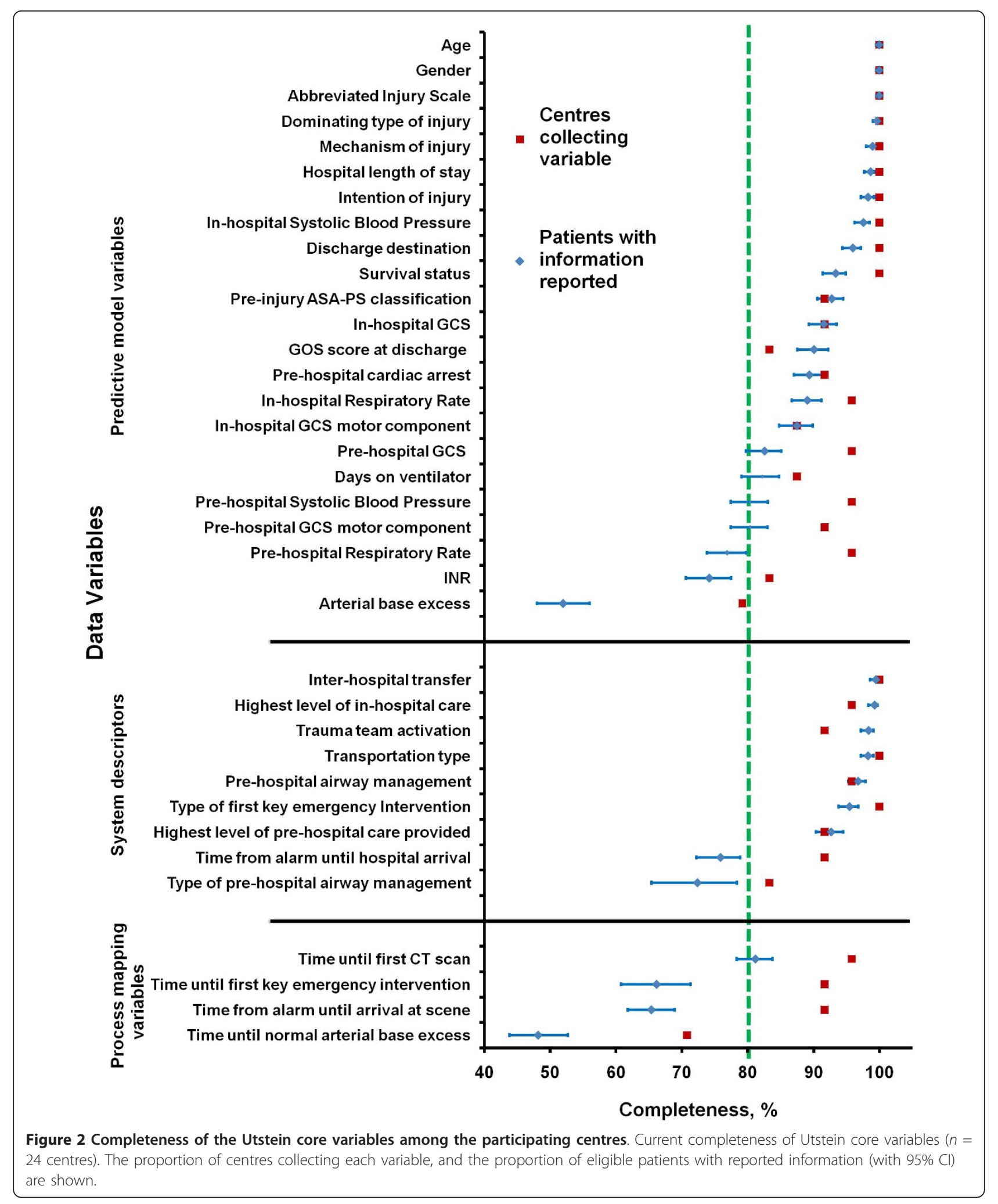




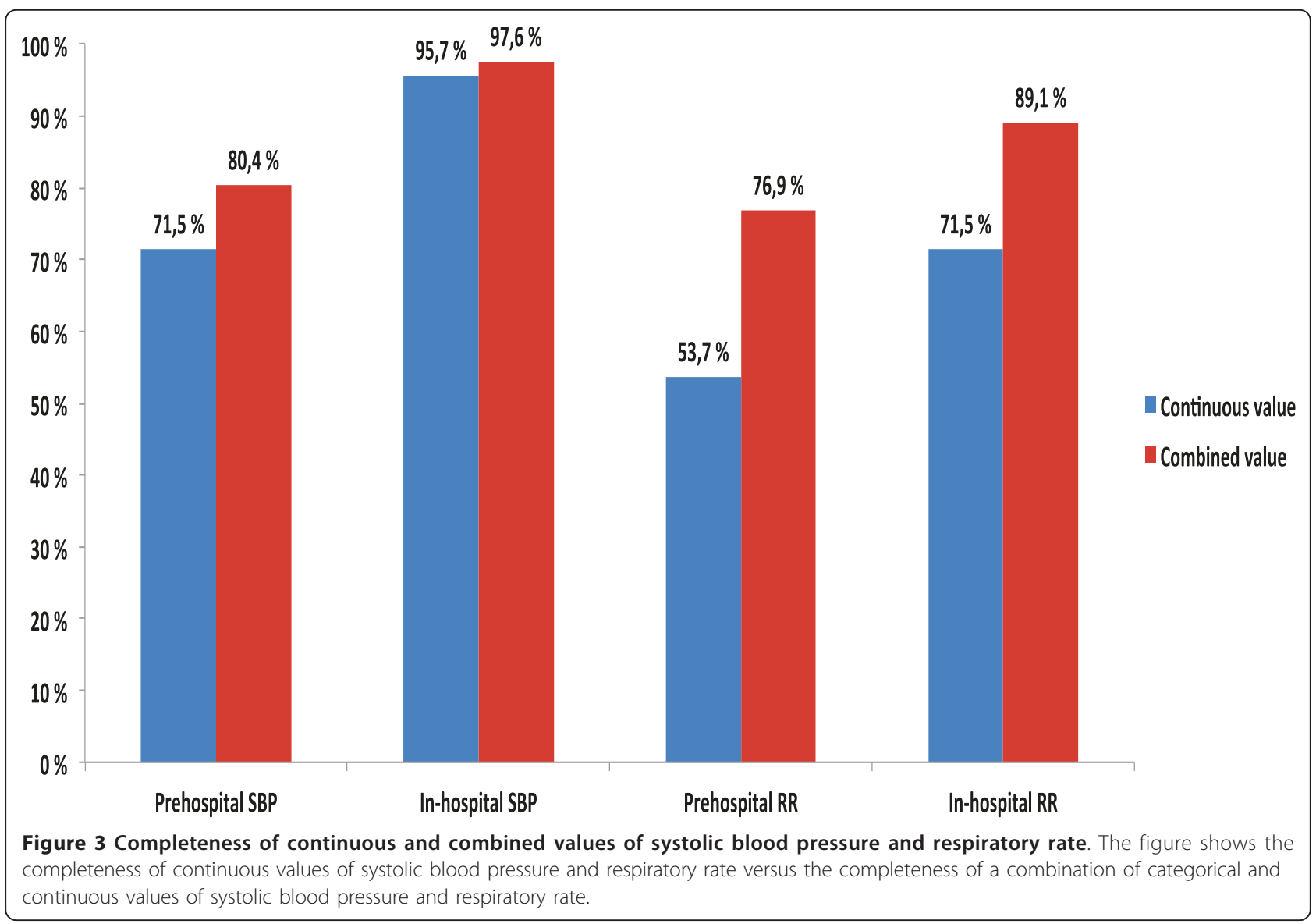

Template, the balance between 'desirability' and 'collectability' of a variable was probably in favour of the former because there were no objective data on 'collectability'. This study has identified variables that are particularly difficult to collect. In particular, the collection of "Arterial Base Excess", and process data like "Time Until Normal Arterial Base Excess" needs to be reconsidered or even excluded from the template, while uniform survival outcome variables and type of AIS coding systems used, should be further agreed upon. Additional studies should review the propriety of some of the variables. Furthermore, the data variables should be evaluated with regard to inter-rater reliability [34].

The template was primarily developed for patients who were directly admitted to a trauma centre. A more complete assessment of the performance of the entire trauma system [35] will need to include transferred patients. Exclusion of transferred patients may strongly influence the results when hospitals with large proportions of transferred patients are included.

In order to further develop an international core dataset, a consensus-driven revision of the Utstein Trauma Template, with representatives from multiple continents, should be initiated. The results of the current study will be valuable for such a revision.

\section{Conclusions}

The study shows that $78 \%$ of the data variables of the Utstein Trauma Template were $>80 \%$ complete. Difficulty with collecting time variables and a lack of uniformity in the use of outcome variables and injury scoring systems across international trauma institutions were found. Overall, the feasibility of collecting most of the core data was demonstrated across several registries and countries.

\section{Key messages}

- The use of the common trauma template was feasible across international registries for the majority of the data variables.

- A lack of uniformity in the use of outcome variables and injury scoring systems across international trauma institutions mandate a need for better standardisation.

- The current results may serve as a stepping-stone towards creation of a European trauma registry. 


\section{Additional material}

Additional file 1: The self-administered questionnaire. This file includes the self-administered questionnaire that was distributed to the participating centres.

Additional file 2: Completeness of the Utstein core variables among the participating centres. The table includes the number of centres collecting a data variable, completeness of patient data from the recording centres, and the number of centres with complete patient data by percentiles.

\section{Abbreviations}

AIS: Abbreviated Injury Scale; IQR: interquartile range; ISS: Injury Severity Score; NISS: New Injury Severity Score; RR: respiratory rate; RTS: Revised Trauma Score; SBP: systolic blood pressure.

\section{Acknowledgements}

The Utstein Trauma Data Collaborators

The following people are acknowledged for their assistance with the data collection:

Australia: The Royal Children's Hospital Melbourne, Victoria: Cameron S. Palmer.

Denmark: Copenhagen University Hospital, Rigshospitalet, Copenhagen: Claus Falck Larsen; Odense University Hospital, Odense: Anni Ellegaard Hansen, Morten Schultz Larsen, Jens M. Lauritsen.

Finland: Meilahti Hospital, Helsinki University Hospital, Helsinki: Piia Hienonen Ari Leppäniemi; Töölö Hospital, Helsinki University Hospital, Helsinki: Lauri Handolin.

Germany: Trauma Registry of the German Society of Trauma Surgery, Cologne: Rolf Lefering

Greece: Asklepeion Voulas General Hospital, Athens: George Giannopoulos. Italy: Azienda Ospedaliera G. Rummo, Benevento: Concetta Pellegrini; Azienda Ospedaliera San Camillo-Forlanini, Roma: Giulia Ranaldi; Azienda Ospedaliera C.T.O./Maria Adelaide, Torino: Teresa Piro.

Lithuania: Vilnius University Children's Hospital, Vilnius: Ruta Kvederiene, Virginija Zilinskaite.

The Netherlands: Academic Medical Center, Amsterdam: J. Carel Goslings, Christiaan Ponsen; VU University Medical Center, Amsterdam: Frank Bloemers, Georgios Giannakopoulos.

Norway: Oslo University Hospital-Ullevål, Oslo: Torsten Eken, Morten Hestnes, Olav Røise, Nils Oddvar Skaga, Petter Andreas Steen; Stavanger University Hospital, Stavanger: Kjetil Søreide, Kjell Tjosevik; St. Olav University Hospital, Trondheim: Oddvar Uleberg.

Portugal: Hospital de Santo António, Centro Hospitalar do Porto, Porto: Ernestina Gomes.

Sweden: Karolinska University Hospital, Solna, Stockholm: Olof Brattström; Södersjukhuset University Hospital, Stockholm: Maaret Castrèn, Rebecka Rubenson Wahlin.

Switzerland: Centre Hospitalier Universitaire Vaudois, Lausanne: Catherine Heim

United Kingdom: The Trauma Audit \& Research Network, University of Manchester, Manchester; Timothy J. Coats, Antoinette Edwards, Thomas Lawrence, Fiona Lecky, Maralyn Woodford.

USA: Denver Health Medical Center, Denver, Colorado: Walter L. Biffl, Craig Gravitz;

Johns Hopkins Hospital Adult Trauma Center, Baltimore, Maryland: Adil H. Haider, Kathy Noll; Regions Hospital, St. Paul, Minnesota and Gillette Children's Hospital, St. Paul, Minnesota: Heidi M. Altamirano, Sandy Brengman, David J. Dries, Michael D. McGonigal. Supervisors

Professor Olav Røise MD PhD, Nils Oddvar Skaga MD PhD and Professor Petter Andreas Steen MD PhD, Oslo University Hospital-Ullevål, Norway are acknowledged for their contributions to planning of the project and supervising KGR

Discussions on completeness thresholds

We thank Professor Douglas G. Altman, Centre for Statistics in Medicine, University of Oxford, UK for valuable considerations on aspects of completeness thresholds.
AIS-to-NISS calculation system

Torsten Eken, MD PhD, Oslo University Hospital-Ullevål, Norway is acknowledged for providing a Microsoft Excel-based AIS-to-NISS conversion system.

Databases

The Italian Trauma Registry is acknowledged for providing the participating Italian hospitals with a trauma registry, and the UK Trauma Audit and Research Network is acknowledged for providing Copenhagen University Hospital with a registration tool. Acknowledgements are also extended to the EpiData Association, Odense, Denmark for providing a database to hospitals without an adequate registry.

Data storage

IT Department, Norwegian Air Ambulance Foundation, Drøbak, Norway. PhD funding

The Norwegian Air Ambulance Foundation, Drøbak, Norway funded the PhD scholarship of Kjetil G. Ringdal, MD.

\section{Author details}

'Department of Research, Norwegian Air Ambulance Foundation, Holterveien 24, N-1440 Drøbak, Norway. ${ }^{2}$ Division of Emergencies and Critical Care, Oslo University Hospital-Ullevål, Kirkeveien 166, N-0450 Oslo, Norway. ${ }^{3}$ Institute of Clinical Medicine, Faculty of Medicine, University of Oslo, Kirkeveien 166, N-0450, Norway. ${ }^{4}$ Department of Surgical Sciences, Faculty of Medicine and Dentistry, University of Bergen, N-5021 Bergen, Norway. ${ }^{5}$ Mathematics Department, School of Computing and Mathematics, Faculty of Natural Sciences, Colin Reeves Building, Keele University, Keele, Staffordshire ST5 5BG, UK. ${ }^{6}$ Orthopaedic Department, Accident Analysis Group, Odense University Hospital, Sdr. Boulevard 29, DK-5000 Odense C, Denmark. ${ }^{7}$ Institute of Public Health, Department of Biostatistics, Faculty of Health Sciences, University of Southern Denmark, Campusvej 55, DK-5230 Odense M, Denmark. ${ }^{8}$ Emergency Medicine Academic Group, Department of Cardiovascular Sciences, University of Leicester, Infirmary Square, Leicester LE1 5WW, UK. 9The Trauma Audit \& Research Network, Clinical Sciences Building, Hope Hospital, Eccles Old Road, Salford M6 8HD, UK. ${ }^{10}$ Trauma Service, The Royal Children's Hospital Melbourne, Flemington Road, Parkville, VIC 3052, Australia. ${ }^{11}$ Institute for Research in Operative Medicine, Faculty of Health, University of Witten/Herdecke, Ostmerheimer Str. 200, Haus 38, 51109 Cologne, Germany. ${ }^{12}$ Trauma Registry of the German Society of Trauma Surgery, Ostmerheimer Str. 200, 51109 Cologne, Germany. ${ }^{13}$ Department of Anaesthesia and ICU, Azienda Ospedaliero-Universitaria di Udine, Piazzale Santa Maria della Misericordia, 33100 Udine, Italy. ${ }^{14}$ Italian National Trauma Registry and Emilia-Romagna Trauma Registry, Department of Clinical Governance, Regional Health Agency, Viale Aldo Moro 21, 40127 Bologna, Italy. ${ }^{15}$ Department of Surgery, Regions Hospital, 640 Jackson Street, St. Paul, MN 55101, USA. ${ }^{16}$ Department of Surgery, University of Minnesota, 420 Delaware Street SE, Minneapolis, MN 55455, USA. ${ }^{17}$ Department of Surgery, Stavanger University Hospital, Armauer Hansens vei 20, N-4011 Stavanger, Norway.

\section{Authors' contributions}

KGR designed the study, prepared and analysed the data, drafted the results, and drafted the manuscript. HML designed the study, assisted in drafting the results, and drafting the manuscript. KS designed the study, assisted in drafting the results, and drafted the manuscript. JMJ designed the study, prepared and analysed the data, assisted in drafting the results, and assisted in drafting the manuscript. JML and CSP assisted in drafting the results, and assisted in drafting the manuscript. TJC, RL, SDP, and DJD assisted in drafting the manuscript. All authors read and approved the final manuscript for publication. The Utstein Trauma Data Collaborators were invited to read and comment on the final manuscript draft.

\section{Authors' information}

KGR, HML, TJC and RL are members of the European Trauma Registry Network's working party for the establishment of a European trauma registry. KGR drafted the Utstein Trauma Template Data Dictionary and is a member of the working party for the establishment of the Norwegian National Trauma Registry. TJC is the chairman of the Trauma Audit and Research Network, UK. CSP chaired the working party of the National Trauma Registry Consortium in Australia and New Zealand for the formulation of a Binational Minimum Dataset (BMDS) and drafted the BMDS Data Dictionary in 2010. RL is co-chairman and statistician of the Trauma 
Registry of the German Society of Trauma Surgery. SDP is the scientific director of the Italian National Trauma Registry.

\section{Competing interests}

The authors declare that they have no competing interests.

Received: 29 May 2011 Revised: 15 July 2011

Accepted: 12 October 2011 Published: 12 October 2011

\section{References}

1. Søreide K: Epidemiology of major trauma. Br J Surg 2009, 96:697-698

2. World Health Organization: The global burden of disease: 2004 update. World Health Organization; Geneva, Switzerland; 2008 [http://www.who.int/ healthinfo/global_burden_disease/2004_report_update/en/index.html].

3. Celso B, Tepas J, Langland-Orban B, Pracht E, Papa L, Lottenberg L, Flint L: A systematic review and meta-analysis comparing outcome of severely injured patients treated in trauma centers following the establishment of trauma systems. J Trauma 2006, 60:371-378, discussion 378.

4. Gabbe BJ, Lecky FE, Bouamra O, Woodford M, Jenks T, Coats TJ, Cameron PA: The effect of an organized trauma system on mortality in major trauma involving serious head injury: a comparison of the United Kingdom and Victoria, Australia. Ann Surg 2011, 253:138-143.

5. Edwards A, Di Bartolomeo S, Chieregato A, Coats T, Della Corte F, Giannoudis P, Gomes E, Groenborg H, Lefering R, Leppäniemi A, Lossius HM, Ortenwal P, Røise O, Rusnak M, Sturms L, Smith M, Bondegaard Thomsen A, Willett K, Woodford M, Yates D, Lecky F: A comparison of European Trauma Registries. The first report from the EuroTARN Group. Resuscitation 2007, 75:286-297.

6. Garthe E: Overview of trauma registries in the United States. J AHIMA 1997, 68:26, 28-32; quiz 33-24..

7. Mann NC, Guice K, Cassidy L, Wright D, Koury J: Are statewide trauma registries comparable? Reaching for a national trauma dataset. Acad Emerg Med 2006, 13:946-953.

8. Owen $\mathrm{JL}$, Bolenbaucher RM, Moore ML: Trauma registry databases: a comparison of data abstraction, interpretation, and entry at two level i trauma centers. J Trauma 1999, 46:1100-1104.

9. Ringdal $\mathrm{KG}$, Lossius HM: Feasibility of comparing core data from existing trauma registries in Scandinavia. Reaching for a Scandinavian major trauma outcome study (MTOS). Scand J Surg 2007, 96:325-331.

10. de Jongh MA, Verhofstad MH, Leenen LP: Accuracy of different survival prediction models in a trauma population. Br J Surg 2010, 97:1805-1813.

11. Nolte E: International Benchmarking of Healthcare Quality: A Review of the Literature. RAND Europe and London School of Hygiene and Tropical Medicine; Cambridge, United Kingdom; 2010 [http://www.rand.org/ randeurope]

12. National Trauma Data Bank: National Trauma Data Standard: Data Dictionary. 2011 [http://www.ntdsdictionary.org/].

13. Leppäniemi A: Trauma systems in Europe. Curr Opin Crit Care 2005, 11:576-579.

14. Kristiansen T, Søreide K, Ringdal KG, Rehn M, Krüger AJ, Reite A, Meling T, Naess PA, Lossius HM: Trauma systems and early management of severe injuries in Scandinavia: review of the current state. Injury 2010, 41:444-452.

15. Ringdal KG, Coats TJ, Lefering R, Di Bartolomeo S, Steen PA, Røise O, Handolin L, Lossius HM: The Utstein template for uniform reporting of data following major trauma: a joint revision by SCANTEM, TARN, DGUTR and RITG. Scand J Trauma Resusc Emerg Med 2008, 16:7.

16. Ringdal KG, Coats TJ, Lefering R, Di Bartolomeo S, Steen PA, Røise O, Handolin L, Castrén M, Christensen EF, Edwards A, Eken T, Gomes E, Hestnes M, Klarin L, Larsen MS, Lauritsen J, Leppäniemi A, Örtenwall P, Skaga NO, Wisborg T, Woodford M, Lossius HM: The Utstein Trauma Template for Uniform Reporting of Data Following Major Trauma: Data Dictionary. 2008. European Trauma Registry Network 2008 [http://www. eurotraumanet/].

17. Coats TJ: A revised Utstein Template for trauma. Emerg Med J 2010, 27:339.

18. von Elm E, Altman DG, Egger M, Pocock SJ, Gotzsche PC Vandenbroucke JP: The Strengthening the Reporting of Observational Studies in Epidemiology (STROBE) statement: guidelines for reporting observational studies. Lancet 2007, 370:1453-1457.
19. Osler T, Baker SP, Long W: A modification of the injury severity score that both improves accuracy and simplifies scoring. J Trauma 1997, 43:922-925, discussion 925-926.

20. Association for the Advancement of Automotive Medicine: Abbreviated Injury Scale (AIS) 2005-Update 2008. Barrington, IL: Association for the Advancement of Automotive Medicine; 2008.

21. Wilson EB: Probable inference, the law of succession, and statistical inference. J Am Stat Assoc 1927, 22:209-212.

22. Champion HR, Sacco WJ, Copes WS, Gann DS, Gennarelli TA, Flanagan ME: A revision of the trauma score. J Trauma 1989, 29:623-629.

23. Arbabi S, Jurkovich GJ, Wahl WL, Franklin GA, Hemmila MR, Taheri PA, Maier RV: A comparison of prehospital and hospital data in trauma patients. J Trauma 2004, 56:1029-1032.

24. Altman DG, Royston P: The cost of dichotomising continuous variables. BMJ 2006, 332:1080.

25. van Walraven C, Hart RG: Leave 'em alone-why continuous variables should be analyzed as such. Neuroepidemiology 2008, 30:138-139.

26. Salottolo K, Settell A, Uribe P, Akin S, Slone DS, O'Neal E, Mains C, Bar-Or D: The impact of the AIS 2005 revision on injury severity scores and clinical outcome measures. Injury 2009, 40:999-1003.

27. Palmer CS, Niggemeyer LE, Charman D: Double coding and mapping using Abbreviated Injury Scale 1998 and 2005: identifying issues for trauma data. Injury 2010, 41:830-836.

28. Palmer CS, Franklyn M: Assessment of the effects and limitations of the 1998 to 2008 Abbreviated Injury Scale map using a large populationbased dataset. Scand J Trauma Resusc Emerg Med 2011, 19:1.

29. Baker SP, O'Neill B, Haddon W Jr, Long WB: The injury severity score: a method for describing patients with multiple injuries and evaluating emergency care. J Trauma 1974, 14:187-196.

30. Palmer CS, Franklyn M, Read-Allsopp C, McLellan S, Niggemeyer LE: Development and validation of a complementary map to enhance the existing 1998 to 2008 Abbreviated Injury Scale map. Scand J Trauma Resusc Emerg Med 2011, 19:29.

31. Skaga NO, Eken T, Jones JM, Steen PA: Different definitions of patient outcome: consequences for performance analysis in trauma. Injury 2008 39:612-622.

32. Jones JM: An approach to the analysis of trauma data having a response variable of death or survival. J Trauma 1995, 38:123-128.

33. Jones JM, Maryosh J, Johnstone S, Templeton J: A multivariate analysis of factors related to the mortality of blunt trauma admissions to the North Staffordshire Hospital Centre. J Trauma 1995, 38:118-122.

34. Streiner DL, Norman GR: Health Measurement Scales. A Practical Guide to Their Development and Use. 4 edition. New York, NY: Oxford University Press Inc; 2008.

35. Lossius HM, Kristiansen T, Ringdal KG, Rehn M: Inter-hospital transfer: the crux of the trauma system, a curse for trauma registries. Scand J Trauma Resusc Emerg Med 2010, 18:15.

\section{doi:10.1186/cc10485}

Cite this article as: Ringdal et al: Collecting core data in severely injured patients using a consensus trauma template: an international multicentre study. Critical Care 2011 15:R237.

\section{Submit your next manuscript to BioMed Central and take full advantage of:}

- Convenient online submission

- Thorough peer review

- No space constraints or color figure charges

- Immediate publication on acceptance

- Inclusion in PubMed, CAS, Scopus and Google Scholar

- Research which is freely available for redistribution 